\title{
In vivo alterations of IFN regulatory factor-1 and PIAS1 protein levels in cystic fibrosis epithelium
}

\author{
Thomas J. Kelley and Heather L. Elmer \\ Department of Pediatrics, Case Western Reserve University, Cleveland, Ohio, USA \\ Address correspondence to: Thomas J. Kelley, Department of Pediatrics, Case Western Reserve University, \\ 8th Floor Biomedical Research Building, 10900 Euclid Avenue, Cleveland, Ohio 44106-4948, USA. \\ Phone: (216) 368-0831; Fax: (216) 368-4223; E-mail: tjk12@po.cwru.edu. \\ Received for publication February 9, 2000, and accepted in revised form June 13, 2000.
}

Inducible nitric oxide synthase-2 (NOS2) expression has been shown to be reduced in cystic fibrosis (CF) epithelial cells. Reduced NOS2 expression is unexpected, given the inflammatory nature of CF airway disease, and is an indication that cell-signaling mechanisms necessary for proper NOS2 regulation are probably altered in $\mathrm{CF}$ epithelium. Therefore, we examined the expression levels of regulatory factors necessary for NOS2 expression in CF epithelium and showed that IFN regulatory factor-1 (IRF-1) is necessary for full NOS2 expression. Mice lacking IRF-1 expression have diminished epithelial NOS2 expression, as well as reduced NO-dependent chloride transport across the nasal epithelia. Furthermore, IRF-1 protein expression is reduced in nasal and intestinal epithelial cells from CF mice, suggesting a possible mechanism for the CF-related reduction of epithelial NOS2 expression. Active signal transducer and activator of transcription-1 (Stat1) is necessary for both NOS2 and IRF-1 expression. We found that protein levels of Stat1 were increased in CF cells, but that the active phosphorylated form of Stat 1 was bound to the protein inhibitor of activated Stat1 (PIAS1). We propose that increased levels of PIAS1 diminish certain cell-signaling pathways, resulting in reduced IRF-1 and NOS2 expression in CF epithelial cells.

J. Clin. Invest. 106:403-410 (2000).

\section{Introduction}

Cystic fibrosis (CF) is a disease caused by mutations in the gene encoding the cystic fibrosis transmembrane conductance regulator (CFTR) (1). CFTR is a protein kinase A-activated chloride channel responsible for the regulation of transepithelial chloride transport in several tissues, including those of the respiratory system, pancreatic ducts, sweat glands, and the intestines (1). CF airway disease is characterized by excessive or altered mucous production, an overzealous inflammatory response, progressive tissue damage, and chronic bacterial infection (1). It is unclear, however, how the loss of a single epithelial chloride channel results in the multitude of symptoms associated with CF airway disease. It has recently been shown that the expression of the inducible form of nitric oxide synthase-2 (NOS2) is reduced in $\mathrm{CF}$ airway epithelium from both human subjects and from murine models of CF (2-4). This reduction in NOS2 expression and subsequent reduction in $\mathrm{NO}$ production has been postulated to play a role in the abnormal regulation of transepithelial sodium absorption observed in CF and in the CF-associated characteristic of susceptibility to bacterial infection $(2,3)$.

Given the inflammatory nature of $\mathrm{CF}$ airway disease, it is unclear why expression of NOS2 would be reduced. Other inflammatory airway diseases such as asthma are associated with increased production of NO (5). Another report demonstrates that the inducible production of the cytokine RANTES is also reduced in CF epithelial cells (6). NOS2 and RANTES are both optimally induced by stimulating NF- $\mathrm{KB}$ and IFN- $\gamma$ pathways, suggesting that reduced expression of these proteins is the result of changes in a common cell-signaling pathway. Although the authors demonstrate a likely role for altered NF- $\kappa B$ activity in the diminished expression of RANTES in CF epithelial cells (6), most reports indicate that NF- $\mathrm{KB}$ activity is either normal or increased in CF epithelium (7). The hypothesis of increased NF- $\mathrm{KB}$ activity in CF epithelial cells is supported by reports demonstrating the hyperinflammatory response characteristic of CF airway disease (8-10). Therefore, we have chosen to focus on components of the IFN- $\gamma$ signaling pathway to determine if alterations in this pathway may be responsible for the observed decrease in NOS2 expression.

The NOS2 promoter has several regulatory components that are essential for complete expression of NOS2, including NF- $\kappa \mathrm{B}, \mathrm{AP}-1$, signal transducer and activator of transcription-1 (Stat1), and IFN regulatory factor-1 (IRF-1) binding sites (11-13). Stat 1 and IRF1 are components of the IFN- $\gamma$ signaling pathway, and both have been shown to influence NOS2 expression. Neither macrophages nor glial cells from IRF-1 knockout mice exhibit cytokine-induced NOS2 expression $(14,15)$. Similarly, Stat 1 has been shown to play an equally important role in induction of NOS2 and IRF1 expression (16-20). The goal of this study is to examine IFN- $\gamma$-dependent signaling components necessary 
for the expression of NOS2 (outlined in Figure 1) in a murine model of CF. We have found that cell-signaling changes appear to be responsible for the paradoxical observation of reduced NOS2 expression in CF epithelial cells. The identification of altered cell-signaling pathways in CF will help clarify the mechanisms responsible for the multitude of symptoms associated with CF airway disease, and hopefully will lead to more effective targets for therapeutic intervention.

\section{Methods}

Mice. Mice lacking CFTR expression (CFTR $\left.{ }^{t m 1 U n c}\right)$, IRF-1 expression (C57BL/6-Irf1 tmIMak), wild-type C57BL/6J mice, and FABP-hcftr mice (CFTR ${ }^{t m I U n c}$-TgN[FABPCFTR]\#Jaw), were obtained from The Jackson Laboratory (Bar Harbor, Maine, USA). CFTR ${ }^{++}$(wild-type) and wild-type heterozygous mice were siblings of $\mathrm{CFTR}^{-/-}$mice. All mice used were between 10 and 12 weeks of age. Mice were fed a liquid diet as described by Eckman et al. (21). Mice were cared for in accordance with Case Western Reserve University Internal Animal Care and Use Committee guidelines by the CF Animal Core Facility.

NOS2-specific immunostaining. Nasal epithelium was excised from wild-type C57BL/6J $\left(I R F-1^{+/+}\right)$mice and C57BL/6-Irf1 tm1Mak (IRF-1 $\left.1^{-/}\right)$mice. This was fixed for 48 hours in $2 \%$ paraformaldehyde and then set in paraffin blocks. Sections $(5 \mu \mathrm{m})$ were deparaffinized, permeabilized in ice-cold methanol for 5 minutes, and placed in $5 \%$ goat serum in PBS for 2 hours. Antibody against NOS2 (Santa Cruz Biotechnology Inc., Santa Cruz, California, USA) was placed on samples, and they were incubated overnight at $4^{\circ} \mathrm{C}$ at a dilution of 1:300 in PBS. Samples were then washed four times in PBS for 10 minutes per wash. Goat anti-rabbit IgG conjugated to alkaline phosphatase was diluted 1:200 in PBS and incubated with samples for 2 hours at $37^{\circ} \mathrm{C}$. Samples were washed as before and stained for 20 minutes in Vector Red from Novocastra Laboratories (Newcastle, United Kingdom) according to the manufacturer's instructions. Slides were counterstained with hematoxylin.

Nasal transepithelial potential difference measurements. Mouse nasal transepithelial potential difference (TEPD) was measured as described previously (22-24). Briefly, an anesthetic composed of $0.4 \mathrm{mg}$ acepromazine, $11 \mathrm{mg}$ ketamine, and $2 \mathrm{mg}$ xylazine per $\mathrm{mL}$ of PBS was administered to each mouse at $200 \mu \mathrm{L}$ per 20 g body weight. PE-10 tubing drawn out to approximately half its original diameter was inserted $2-3 \mathrm{~mm}$ into the nostril of the mouse. Perfusion was performed with a Razel A-99 syringe pump (Razel Scientific Instruments Inc., Stamford, Connecticut, USA) at a rate of approximately $7 \mu \mathrm{L} / \mathrm{min}$. Ringer's solutions used were chloride-replete HEPES-buffered Ringer's containing HEPES $(10 \mathrm{~mm})$ at $\mathrm{pH} 7.4, \mathrm{NaCl}(138 \mathrm{mM})$, $\mathrm{KCl}(5 \mathrm{mM}), \mathrm{Na}_{2} \mathrm{HPO}_{4}(2.5 \mathrm{mM}), \mathrm{CaCl}_{2}(1.8 \mathrm{mM})$, $\mathrm{MgSO}_{4}(1.0 \mathrm{mM})$; and chloride-free HEPES-buffered Ringer's containing HEPES $(10 \mathrm{mM})$ at $\mathrm{pH} 7.4$, sodium gluconate $(138 \mathrm{mM})$, potassium gluconate $(5 \mathrm{mM})$, $\mathrm{Na}_{2} \mathrm{HPO}_{4}(2.5 \mathrm{mM})$, hemicalcium gluconate $(3.6 \mathrm{mM})$, and $\mathrm{MgSO}_{4}(1.0 \mathrm{mM})$ (all chemicals were from Sigma Chemical Co., St. Louis, Missouri, USA). Mice were exposed initially to chloride-replete Ringer's containing amiloride (100 $\mu \mathrm{M}$; Sigma Chemical Co.) until a plateau was achieved. The perfusion solution was then changed to a chloride-free Ringer's solution also containing amiloride $(100 \mu \mathrm{M})$, and perfusion continued for up to 3 minutes.

Immunoprecipitation and Western immunoblotting. Antibodies against protein inhibitor of activated Stat 1 (PIAS1) (goat), IRF-1 (rabbit), Stat1 (mouse), p-Stat1 (mouse), phosphotyrosine-agarose conjugate (mouse), and protein $\mathrm{A} / \mathrm{G}$ plus agarose conjugate were obtained from Santa Cruz Biotechnology Inc. Protein samples were prepared by homogenizing either excised nasal epithelium or epithelial tissue scraped from sections of mouse ileum in lysis buffer consisting of $50 \mathrm{mM}$ Tris at $\mathrm{pH} 7.5,1 \%$ Triton X-100, $100 \mathrm{mM} \mathrm{NaCl}, 50 \mathrm{mM} \mathrm{NaF}$, $200 \mu \mathrm{M} \mathrm{Na} V_{3} \mathrm{VO}_{4}$, and $10 \mu \mathrm{g} / \mathrm{mL}$ each of pepstatin and leupeptin on ice. Protein concentration of samples was measured using the Dc Protein Assay system from BioRad Laboratories Inc. (Hercules, California, USA). Immunoprecipitation experiments using either antiPIAS1 or anti-phosphotyrosine antibodies were performed with $150 \mu \mathrm{g}$ protein from each sample; samples were incubated overnight with constant mixing at $4^{\circ} \mathrm{C}$. Proteins were separated using SDS-PAGE. Samples were transferred to Immobilon-P membrane (Millipore Corp., Bedford, Massachusetts, USA) using a Trans-Blot SD semi-dry transfer cell from Bio-Rad Laboratories Inc. Blots were blocked in PBS consisting of $\mathrm{NaCl}(138 \mathrm{mM})$, $\mathrm{Na}_{2} \mathrm{HPO}_{4}(15 \mathrm{mM}), \mathrm{KCl}(1.5 \mathrm{mM})$, and $\mathrm{KH}_{2} \mathrm{PO}_{4}(2.5$ $\mathrm{mM}$ ) containing $5 \%$ nonfat dehydrated milk and $0.1 \%$ Tween-20 (Sigma Chemical Co.). Blots were incubated for 1-2 hours at room temperature in PBS with primary antibody (1:800 to 1:1,000 dilution) and then washed three times in PBS with $0.1 \%$ Tween-20. Blots were then incubated with secondary antibody conjugated to horseradish peroxidase (1:4,000 dilution; Sigma Chemical Co.) for 1 hour at room temperature and then washed again as described. Signal was visualized by incubating with SuperSignal chemiluminescent substrate (Pierce Chemical Co., Rockford Illinois, USA) and exposing the membrane to Kodak scientific imaging film (Eastman Kodak Co., Rochester, New York, USA). Quantification of protein expression was accomplished by densitometry using Kodak Digital Science 1D software. Relative density units refer to mean pixel density.

Cell transfection and luciferase assay. 9/ $\mathrm{HTEO}^{-}$cells overexpressing the CFTR R domain (pCEPRF) and mocktransfected 9/HTEo cells (pCEP2) were a generous gift from the lab of Pamela B. Davis, Case Western Reserve University. Cells were cared for as described previously (25). Briefly, $9 / \mathrm{HTEo}^{-}$cells were grown at $37^{\circ} \mathrm{C}$ in $95 \%$ $\mathrm{O}_{2}$ and $5 \% \mathrm{CO}_{2}$ to confluence in DMEM with $10 \% \mathrm{FBS}$, $2.5 \mathrm{mM}$ L-glutamine, and $150 \mu \mathrm{g} / \mathrm{mL}$ hygromycin on Vitrogen-coated, 30-mm tissue culture plates (Collagen Biomaterials, Palo Alto, California, USA). Cells were seeded at $5.0 \times 10^{5}$ cells per well in 6 -well culture dish- 
es 24 hours before transfection. For each transfection, $1 \mu \mathrm{g}$ each of pEGFPN1 (CLONTECH Laboratories, Palo Alto, California, USA) and pGAS-Luc (Stratagene, La Jolla, California, USA) were placed into $100 \mu \mathrm{L}$ of serum-free culture medium, along with $8 \mu \mathrm{L}$ Lipofectamine (GIBCO BRL, Gaithersburg, Maryland, USA). The two solutions were mixed at room temperature and were allowed to incubate for up to 40 minutes. After rinsing, cells were placed in $0.8 \mathrm{~mL}$ of serum-free culture medium. The DNA-liposome mixture $(0.2 \mathrm{~mL})$ was added to each well and mixed gently. Cells were incubated with the complex for 5 hours at $37^{\circ} \mathrm{C}$ in $95 \%$ $\mathrm{O}_{2}$ and $5 \% \mathrm{CO}_{2}$. After incubation, $1 \mathrm{~mL}$ of growth medium (20\% serum) was added to each well to a final concentration of $10 \%$ serum; this was incubated overnight. Serum-free medium was placed in each well 2 hours before stimulation (except for serum-stimulated cells). IFN- $\gamma$ was then added at concentrations of 10 , 25 , and 100 units $/ \mathrm{mL}$, and the mixture was incubated for 5 hours at $37^{\circ} \mathrm{C}$ in $95 \% \mathrm{O}_{2}$ and $5 \% \mathrm{CO}_{2}$. Cells were then lysed and assayed for luciferase activity using Luciferase Assay Systems according to the manufacturer's instructions (Promega Corp., Madison, Wisconsin, USA). Luciferase activity was determined on a Tropix (Bedford, Massachusetts, USA) automatic injection luminometer. Green fluorescent protein (GFP) fluorescence was also determined in triplicate from cell lysates on an Fmax fluorometer from Molecular Devices Corp. (Sunnyvale, California, USA). Luciferase activity was normalized for transfection efficiency by reporting data as a ratio of relative light units (RLU) to relative fluorescence units (RFU) for each well.

\section{Results}

IRF-1 is a necessary factor for constitutive epithelial NOS2 expression. It has already been shown that inducible expression of NOS2 is diminished in macrophages and glial cells isolated from mice lacking IRF-1 expression (IRF-1 $1^{-/-}$mice) $(14,15)$. We examined NOS2 expression in excised nasal epithelium from C57BL/6J mice and $I R F-1^{-1-}$ mice with the same $\mathrm{C} 57 \mathrm{BL} / 6 \mathrm{~J}$ background to determine if IRF-1 expression is necessary for constitutive epithelial NOS2 production. We have shown previously that $\mathrm{NOS}^{-{ }^{--}}$mice with a C57BL/6J background lack NOS2 staining, demonstrating the lack of nonspecific binding of the NOS2 antibody in mice with the C57BL/6J background (3). NOS2-specific immunostaining revealed that normal C57BL/6J mice express detectable NOS2 in nasal epithelium, but that this NOS2 expression is reduced in $I R F-1^{-/-}$mice (Figure 2). The attenuated level of NOS2 expression in the nasal epithelium of $I R F-1^{-/-}$mice demonstrates dependence on IRF-1 for constitutive NOS2 expression, and its expression should be explored in CF epithelium.

IRF-1-mediated regulation of transepithelial chloride transport. To establish a functional effect of reduced IRF-1 expression on epithelial NO production, we examined the chloride-free response using the nasal TEPD assay in $I R F-1^{-1-}$ mice and normal C57BL/6J mice. The chlo- ride-free response was measured by perfusing the nasal passage of the mice with a chloride-free Ringer's solution in the presence of the sodium-transport inhibitor amiloride. Absence of chloride in the Ringer's solution establishes a driving force for the secretion of chloride across the nasal epithelium into the lumen. This response can be considered a measurement of basal chloride transport properties of the epithelium in that no chloride secretory agonists are present. We have shown previously that NOS2-/- mice have a significantly reduced chloride-free response compared with normal C57BL/6J mice, but this response returns to near wild-type levels when the NO donor sodium nitroprusside (SNP) is added to the perfusion solution (4). These data point to a role for NO in regulating basal transepithelial chloride transport. This experiment was repeated using $I R F-1^{-/-}$mice. Wild-type C57BL/6J mice show a $-9.9 \pm 1.2 \mathrm{mV}(n=6)$ hyperpolarization in response to chloride-free Ringer's after 3 minutes, indicating robust chloride secretion across the nasal epithelium. IRF-1 $1^{-/-}$mice, however, showed a hyperpolarization of only $-2.1 \pm 1.1 \mathrm{mV}(n=8)$ under the same conditions, indicating reduced endogenous stimulus for chloride transport that was essentially identical to that observed with the NOS2 mice (Figure 3) (4). When the NO donor SNP $(100 \mu \mathrm{M})$ is added to the perfusion solution, the chloride secretory response increases to a $-8.5 \pm 1.0 \mathrm{mV}(n=5)$ hyperpolarization in IRF-1/-- mice. These data show a functional consequence of attenuated NO production due to the elimination of IRF-1 expression. Consistent with previous findings, IRF-1 is a factor essential for the proper regulation of epithelial $\mathrm{NO}$ production in vivo.

In vivo constitutive IRF-1 expression in $C F$ and non-CF epithelium. Examination of IRF-1 expression levels from excised nasal epithelium from $\mathrm{CFTR}^{+/-}$mice and $\mathrm{CFTR}^{-/-}$mice revealed that IRF-1 levels were significantly reduced in $\mathrm{CFTR}^{-/-}$nasal epithelium compared with those in epithelium from wild-type heterozygous mice (Figure 4a). However, a high-molecular-weight band that was recognized by the IRF-1 antibody did appear in some, but not all, of the CFTR-/- nasal

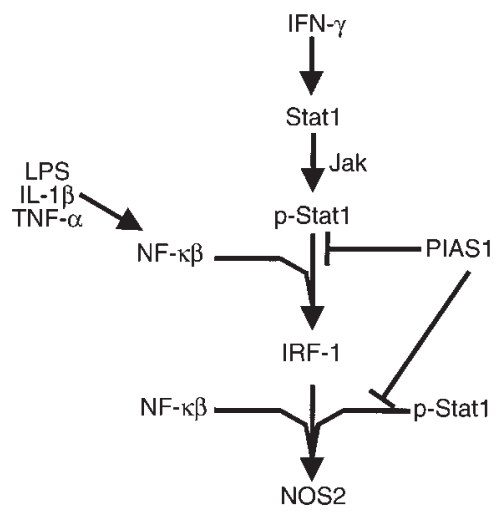

Figure 1

Cell-signaling components involved in the regulation of NOS2 expression. 


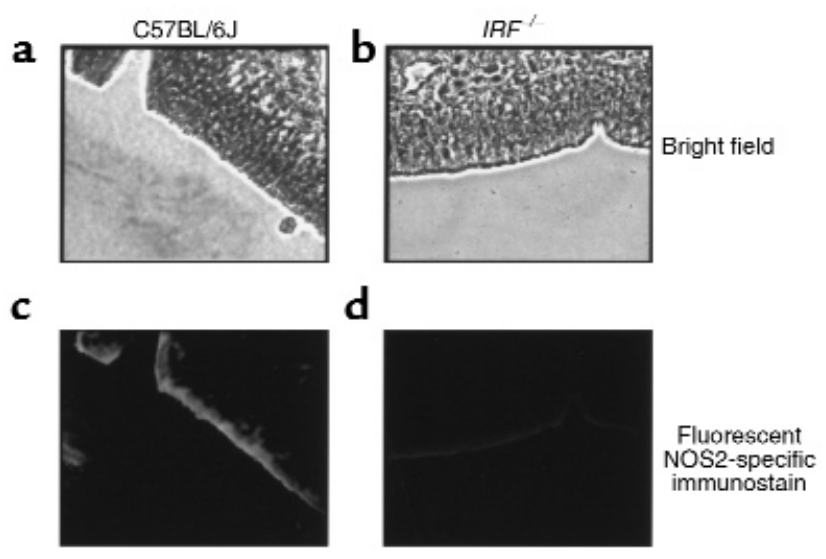

Figure 2

NOS2-specific immunostaining in excised murine nasal epithelial tissue. Bright-field view of nasal epithelial tissue from wild-type C57BL/6J mice (a) and IRF-1-/- mice (b). NOS2-specific fluorescent immunostaining from wild-type C57BL/6J mice (c) and IRF-1-/- mice (d). IRF-1-/- mice have a C57BL/6J background. Images are representative of results obtained with four mice of each genotype. Samples processed identically but not incubated with anti-NOS2 antibody showed no staining (data not shown). Objective images $(\times 40)$.

epithelial samples (not shown). The presence of this band in only some preparations of nasal epithelium inclines us to believe it is a contaminant, but the significance of this high-molecular-weight immunoreactive band in the CF nasal epithelial samples will have to be determined. The figure presents results from three separate $\mathrm{CFTR}^{+/-}$mice and three separate $\mathrm{CFTR}^{-/-}$ mice. Densitometry results are representative of eight wild-type mice and seven CF mice tested. IRF-1 expression is significantly lower in $C F T R^{-/-}$mice, with a $P$ value of 0.002 .

We have shown that constitutive NOS2 expression is dependent on the presence of functional CFTR in epithelial cells from excised ileum and nasal tissues (26). To determine whether IRF-1 and NOS2 expression are influenced by similar CFTR-dependent regulatory mechanisms, we examined IRF-1 expression in the same mouse models used previously to study NOS2 expression. Three groups of mice were examined for IRF-1 expression in epithelial cells excised from the ileum. These groups consisted of $\mathrm{CFTR}^{+/-}$ mice, $\mathrm{CFTR}^{-/-}$mice, and additional $\mathrm{CFTR}^{-/-}$mice that express human CFTR specifically in intestinal epithelium driven by the fatty-acid binding protein (FABP) promoter (FABP-hcftr mice) $(26,27)$. IRF-1 is constitutively expressed in the ileum of $\mathrm{CFTR}^{+/-}$mice and FABP-hcftr mice, but is not expressed in tissue isolated from $\mathrm{CFTR}^{-/-}$mice (Figure 4c). These data demonstrate the same CFTR-dependent IRF-1 expression that we demonstrated previously in the case of NOS2 expression (26). The reintroduction of functional CFTR into the mouse intestinal epithelium restores the expression of IRF-1. Densitometric analysis of protein expression reveals no significant difference in IRF-
1 expression between wild-type and FABP-hcftr mouse ileum, but shows a significant reduction in the ileum of CFTR ${ }^{-/-}$mice.

Stat 1 and PIAS1 expression in CF and non-CF murine epithelium. Our data indicate that expression of NOS2 and IRF1 are diminished in CF epithelial cells by a mechanism related to diminished CFTR expression or function. Because NOS2 and IRF-1 expression are dependent on the activation of Stat1, we examined expression levels of Stat1 in $\mathrm{CF}$ and non-CF mouse nasal epithelium. Stat 1 protein levels were found to be overexpressed in excised nasal epithelium from $\mathrm{CFTR}^{-/-}$mice compared with $\mathrm{CFTR}^{+/+}$ mice (Figure 5a), which is surprising in light of the apparent reduction of IRF-1 expression in CF epithelium.

The apparent reduction of IRF-1 and NOS2 expression suggests that there is an interruption in normal Stat 1 signaling. Because Stat 1 levels are increased, we also examined expression levels of the known Stat1 inhibitor PIAS1. PIAS1, also identified as Gu RNA helicase-II binding protein (GBP) (28), has been shown to bind to Stat 1 and to specifically inhibit Stat1-mediated signaling events (29). PIAS1 levels were elevated in excised nasal epithelium from $\mathrm{CFTR}^{-/-}$mice, but were barely detectable in $\mathrm{CFTR}^{+/-}$and $\mathrm{CFTR}^{+/+}$mice (Figure $5 \mathrm{c})$. We have shown previously that $\mathrm{NO}$ production and NOS2 mRNA expression is reduced in a 9/HTEo cell model of CF (26). Mock-transfected 9/HTEo ${ }^{-}$cells (pCEP2) serve as wild-type cells, whereas cells transfected to overexpress the CFTR R domain (PCEPRF) lack cAMP-mediated chloride secretion and have several CF-like phenotypes $(7,25)$. PIAS1 levels were found

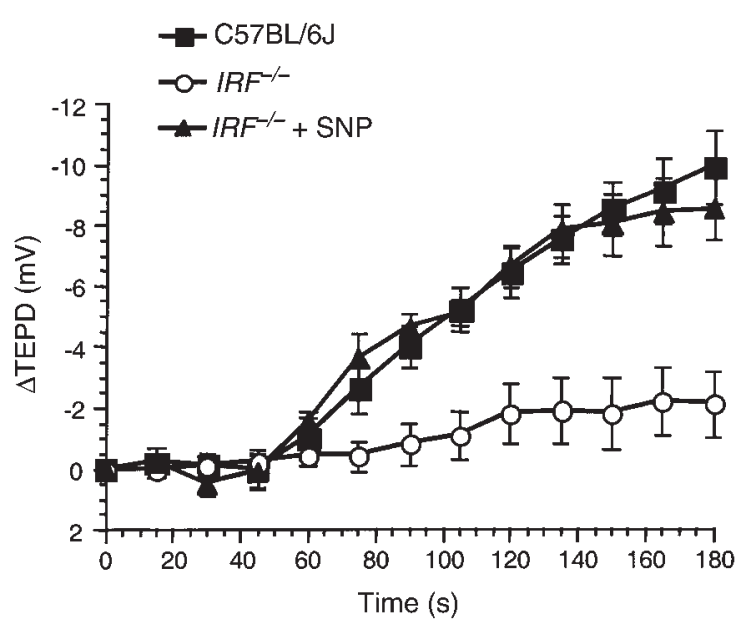

Figure 3

Murine nasal transepithelial chloride transport is partially IRF-1 dependent. Changes in TEPD in response to perfusion with chloridefree Ringer's solution were measured in wild-type C57BL/6J mice (filled squares; $n=6$ ), IRF-1-/- mice (open circles; $n=8$ ), and $I R F-1^{-1-}$ mice with SNP $(100 \mu \mathrm{M})$ added to the perfusion solution (filled triangles; $n=5$ ). Error bars represent SEM. Time zero refers to the point at which the perfusion solution was changed to chloride-free Ringer's (this was done when a plateau value was reached in chloride-replete Ringer's containing amiloride). Amiloride $(100 \mu \mathrm{M})$ was present in all perfusion solutions. 
a

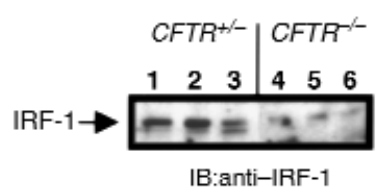

IB:anti-IRF-1

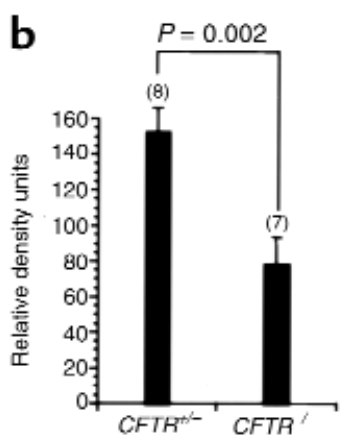

d

c

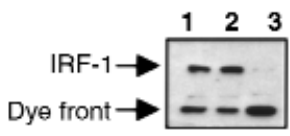

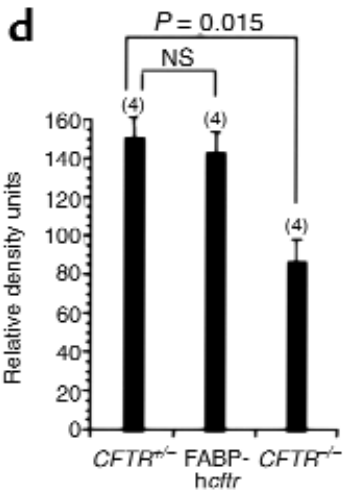

Figure 4

IRF-1 expression in CF and non-CF epithelial tissue. (a) Representative blot of IRF-1 protein expression in excised nasal epithelium from CFTR ${ }^{+/-}$mice (lanes 1-3) and CFTR ${ }^{-/-}$mice (lanes 4-6). Each lane represents tissue isolated from individual mice. (c) IRF-1 expression in excised epithelial tissue from sections of ileum isolated from a CFTR ${ }^{+/-}$mouse (lane 1), an FABP-hcftr mouse (lane 2), and a CFTR $R^{-1-}$ mouse (lane 3 ). (b and $\mathbf{d}$ ) Densitometric analysis of IRF1 -specific immunoblots. Number of samples $(n)$ is shown in parentheses above each bar. Significance determined by Student's $t$ test. Error bars represent SEM. NS, no significant difference between these two values. IB, immunoblot.

to be significantly higher in the CF-phenotype cells (pCEPRF) than in the wild-type phenotype cells (pCEP2) (Figure 5e). Differences in protein expression were quantified by densitometry. Significantly higher expression of both Stat 1 and PIAS1 proteins was found in CF samples (Figure 5, b, d, and f). Increased expression of PIAS1 raises the possibility that Stat 1 signaling may be less than optimal in CF epithelium despite the presence of increased levels of Stat 1 protein.

PIAS1-bound Stat1 in CF and non-CF mouse nasal epitheli$u m$. To address functional differences associated with increased PIAS1 levels in CF epithelium, we examined levels of free and PIAS1-bound Stat1. Stat1 is activated by phosphorylation via the Janus kinase (Jak) family, leading to Stat 1 nuclear translocation, dimerization, and DNA binding. PIAS1 binds to phosphorylated Stat1, preventing dimerization and DNA binding (29). We assessed differences in anti-phosphotyrosine-accessible Stat 1 , and Stat 1 that is apparently associated with PIAS1 in $\mathrm{CF}$ and non-CF cells. Immunoprecipitation with anti- phosphotyrosine antibodies revealed a lack of $\mathrm{p}$-Stat 1 in samples of nasal epithelium from $\mathrm{CFTR}^{-1-}$ mice, whereas Stat 1 levels were clearly detectable in $\mathrm{CFTR}^{+/-}$mouse nasal tissue (Figure 6a). Although PIAS1 is not reported to bind directly to the phosphorylated site of Stat1, we postulated that perhaps PIAS1 binding to $\mathrm{p}$-Stat 1 could interfere with anti-phosphotyrosine antibody binding to p-Stat1. We therefore immunoprecipitated with antiPIAS1 and examined precipitated samples for associated Stat 1 to determine if $\mathrm{p}$-Stat 1 was interacting with increased levels of PIAS1 in CF epithelial cells. Although more PIAS1 was isolated in CF samples by immunopre- $\mathbf{a}$
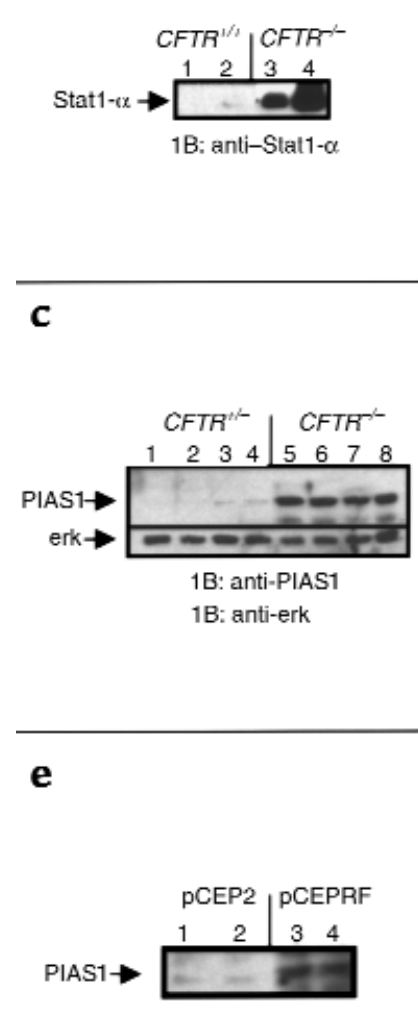

1B: anti-PIAS1 b

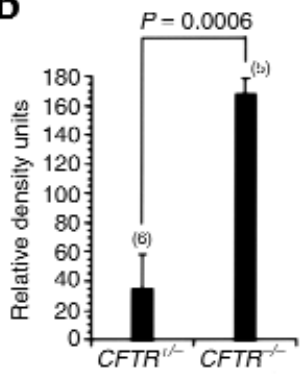

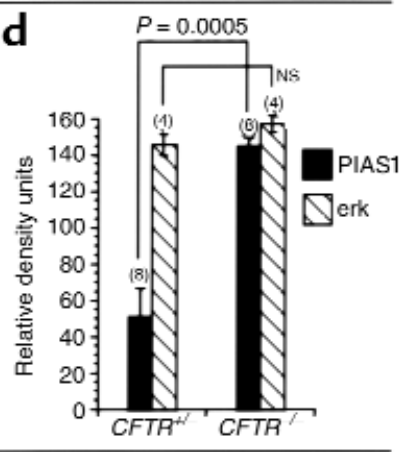

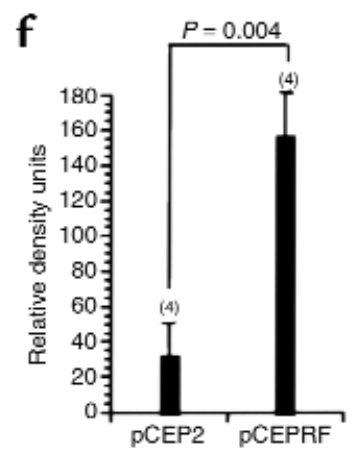

Figure 5

Stat 1 and PIAS1 expression in CF and non-CF model systems. (a) Representative blot of Stat 1 protein expression in excised nasal epithelium from $\mathrm{CFTR}^{+/+}$mice (lanes 1 and 2) and $\mathrm{CFTR}^{-/-}$mice (lanes 3 and 4). Each lane represents tissue isolated from individual mice. (c) PIAS1 expression in excised nasal epithelial tissue from CFTR ${ }^{+-}$mice (lanes 1-4) and CFTR ${ }^{-1-}$ mice (lanes 5-8). This blot was reprobed for erk as a control for protein loading. (e) PIAS1 expression in 9/HTEo ${ }^{-}$pCEP2 control cells (lanes 1 and 2 ) and CF-phenotype 9/HTEo ${ }^{-}$pCEPRF cells (lanes 3 and 4 ). (b, d, f) Densitometric analysis of Stat1, PIAS1, and erk expression. Number of samples $(n)$ is shown in parentheses above each bar. Significance determined by Student's $t$ test. Error bars represent SEM. 
a

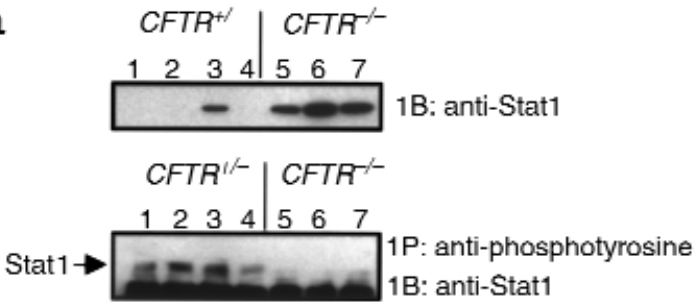

b

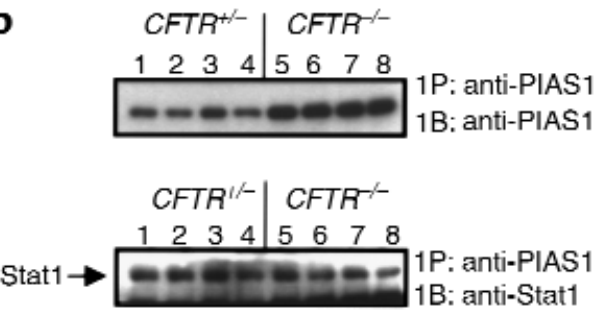

\section{Figure 6}

Levels of free and PIAS1-bound Stat 1 in CF and non-CF mouse nasal epithelial tissue. (a) Upper panel: Representative blot of Stat 1 protein expression in excised nasal epithelium from $\mathrm{CFTR}^{+/-}$mice (lanes 1-4) and CFTR ${ }^{-1-}$ mice (lanes 5-7). Lower panel: Free p-Stat1 levels in the same samples as determined by immunoprecipitation with anti-phosphotyrosine and probing with anti-Stat1. Each lane represents tissue isolated from individual mice. (b) Upper panel: Levels of PIAS1 in excised nasal epithelium from $\mathrm{CFTR}^{+/-}$mice (lanes 1-4) and CFTR ${ }^{-1-}$ mice (lanes 5-8) as determined by immunoprecipitation with anti-PIAS1 and probing with the same antibody. Lower panel: The same immunoprecipitates shown in the upper panel, probed for Stat1 levels. IP, immunoprecipitate; IB, immunoblot.

cipitation with anti-PIAS1 as expected, similar levels of Stat 1 were found in samples from both $\mathrm{CFTR}^{+-}$and $\mathrm{CFTR}^{-1}$ mouse nasal epithelium (Figure 6b). To determine if Stat 1 bound to PIAS1 was indeed phosphorylated, we performed the same experiment of immunoprecipitating PIAS1 and probing the blot with an antibody specific for the phosphorylated form of Stat1. Both CF and non-CF mouse nasal epithelial samples and the 9/HTEo ${ }^{-}$PCEP2 and pCEPRF cells were tested (Figure 7 , a and b, respectively). Phosphorylated Stat1 was bound to PIAS1 in all of the samples. These data demonstrate that p-Stat 1 is present in CF cells, but the majority of available active p-Stat 1 is apparently bound to PIAS1. This observation is true both in vivo in mouse CF epithelial cells and in the CF cell model 9/HTEo pCEP2 $^{-}$ and PCEPRF cells. Increased PIAS1 levels may interfere with full cellular Stat 1 signaling.

Stat1-mediated signaling in 9/HTE $0^{-}$pCEP2 and $p C E P R F$ cells. The CF-phenotype 9/HTEo- pCEPRF cells reflected the same decrease of NOS2 expression (26), the same increase of PIAS1 protein expression (Figure 5), and the same PIAS1-mediated binding of p-Stat 1 (Figure 6) seen in CF mouse nasal epithelium, compared with respective wild-type controls. Therefore, the ability of these cells to carry out Stat1-mediated signaling was tested.
Activated Stat 1 specifically binds to the IFN- $\gamma$-activated site (GAS) to mediate gene transcription, so the luciferase reporter construct pGAS-Luc was transfected into control pCEP2 and CF-phenotype pCEPRF cells. Cells were treated with $0,10,25$, or 100 units $/ \mathrm{mL}$ of recombinant human IFN- $\gamma$ for 5 hours in serum-free media (Figure 8). The CF-phenotype pCEPRF cells had significantly lower basal luciferase activity and significantly lower luciferase activity at each tested concentration of IFN- $\gamma$ than was observed in the control PCEP2 cells. IFN- $\gamma$ also significantly stimulated luciferase activity at each concentration tested in PCEP2 cells, whereas only the two higher concentrations of IFN- $\gamma$ had a measurable effect in the pCEPRF cells. The effect of serum on stimulated luciferase activity was also tested in PCEP2 and pCEPRF cells. Significant stimulation of luciferase activity was observed in the control 9/HTEo ${ }^{-}$PCEP2 cells, but no stimulation occurred in the CF-phenotype pCEPRF cells. Data were normalized for transfection efficiency by measuring GFP fluorescence mediated by cotransfection with pEGFPN1 as described in Methods, and are presented as a ratio of RLU to RFU. These data demonstrate that there is an inherent decrease in Stat1mediated signaling in the CF-phenotype 9/HTEo pCEPRF cells that is consistent with an increase in the expression of PIAS1 protein.

\section{Discussion}

The observation that NOS2 expression is reduced in CF epithelial cells of both mouse and human origin indicates that underlying cell-signaling abnormalities
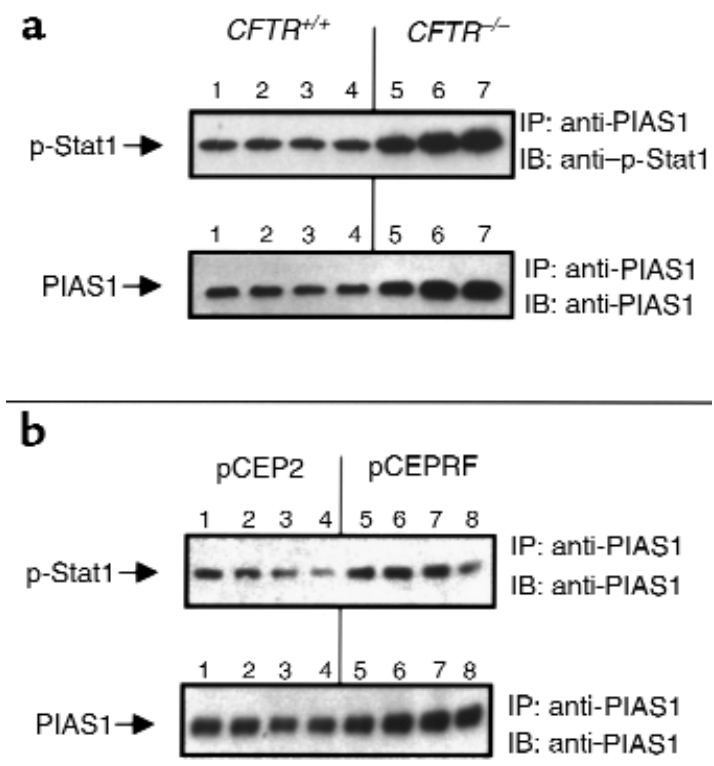

\section{Figure 7}

Levels of p-Stat 1 coprecipitated with PIAS1. To determine if Stat 1 bound to PIAS1 was phosphorylated, PIAS1 was immunoprecipitated from nasal epithelial extracts from four CFTR ${ }^{+/+}$and three CFTR $R^{-/-}$ mice (a), and from four different preparations of 9/HTEo- PCEP2 and PCEPRF cells (b). Precipitates were blotted and probed for immunoreactivity with both anti-PIAS1 and anti-p-Stat 1 antibodies. 


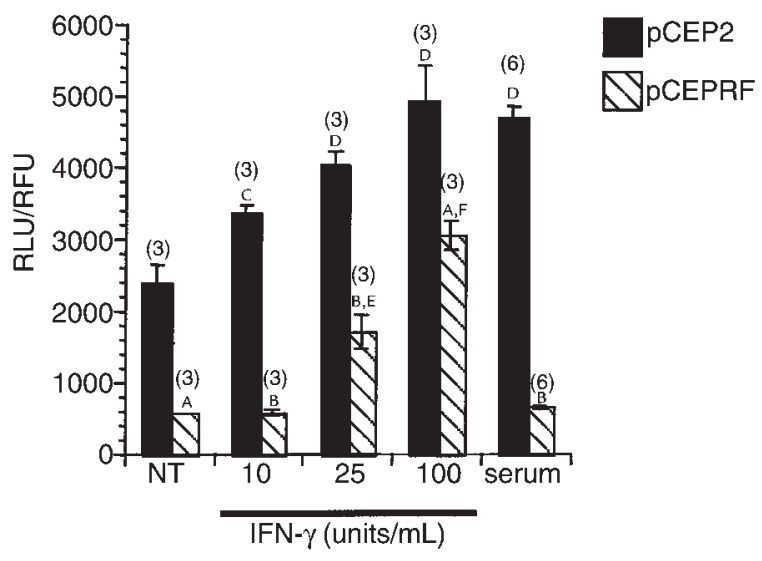

\section{Figure 8}

Stat 1 -mediated cell signaling in 9/HTEo ${ }^{-}$PCEP2 and pCEPRF cells. Cells were cotransfected with pGAS-Luc and pEGFPN1 and stimulated with either 10,25 , or 100 units/mL IFN- $\gamma$ or with $10 \%$ serum as described. Data were normalized for transfection efficiency as measured by GFP expression, and are presented as a ratio of relative light units (RLU) to relative fluorescence units (RFU). The number of experiments $(n)$ is shown in parentheses above each bar. Error bars represent SEM. ${ }^{A} P<0.001 ;{ }^{B} P<0.0001$, pCEP2 vs. PCEPRF cells under each experimental condition, by Student's $t$ test. ${ }^{C, D}$ Significant stimulation of luciferase activity in PCEP2 cells compared with untreated (NT) pCEP2 cells as measured by Duncan's multiple range test ( ${ }^{C} P<$ $0.01 ; \mathrm{D} P<0.0001)$. E,FSignificant stimulation of luciferase activity in pCEPRF cells compared with untreated (NT) pCEPRF cells, as measured by Duncan's multiple range test $\left({ }^{\mathrm{E}} P<0.01 ;{ }^{\mathrm{F}} P<0.0001\right)$.

are present in CF. Both NF- $\mathrm{kB}$ - and IFN- $\boldsymbol{\gamma}$-dependent signaling components are necessary for full NOS2 expression. We decided to explore components of the IFN- $\gamma$ signaling pathway to determine if CF-related changes in this pathway could account for the diminished expression of NOS2 in CF epithelium.

We show that IRF-1 is a necessary factor for functional NO production in murine epithelial cells, and that IRF-1 protein expression is measurably reduced, although not absent, in murine CF nasal epithelial cells. Reduced IRF-1 expression is also dependent on the presence of CFTR, as demonstrated by the use of the FABPhcftr mouse model that exhibits tissue-specific expression of human CFTR in intestinal epithelial cells. Correction of the CFTR defect restores the expression of IRF-1 in mouse intestinal epithelium. These results are consistent with the observation of CFTR-dependent expression of NOS2 using the same model system (26).

Because both NOS2 and IRF-1 have Stat1-reactive elements in their promoters, we examined Stat 1 expression levels. Surprisingly, we found a significant increase in Stat 1 protein expression in CF samples. However, it was also found that protein levels of the Stat1-specific inhibitor PIAS1 are elevated in CF mouse nasal epithelium. As shown in Figure 6, this increase in PIAS1 expression apparently limits access of anti-phosphotyrosine antibodies to $\mathrm{p}$-Stat 1 , a finding that may reflect a reduction in p-Stat 1 levels available for signaling functions. The exact nature of the impact of increased PIAS1 expression on the relative levels of available and unavailable p-Stat 1 requires further exploration.

We have established in an in vivo model of CF that indicates that the immediate cause of reduced NOS2 expression in CF epithelial cells probably involves reduced levels of IRF-1. Reduced IRF-1 levels appear to be related to a reduction in levels of free p-Stat 1 as it becomes bound to the Stat1 inhibitor PIAS1. A significant question that remains to be answered is how PIAS1 expression is influenced by a loss of CFTR function. PIAS1 is related to the Stat 3 inhibitory protein PIAS3. Both PIAS1 and PIAS3 have been identified previously as proteins associated with other functions. PIAS1 was initially identified as GBP, influencing the function of Gu RNA helicase II by modulating proteolysis $(28,29)$. PIAS3 has been found to be homologous to a potassium channel-activating protein, KChAP (30). KChAP has been shown to increase the expression of Kv2 channels and to enhance the current amplitudes of these channels (30). However, there is cross-talk between ion transport regulation and the PIAS family of proteins, and the relationship between CFTR function and the PIAS proteins is currently being explored. Also being explored is the extent of Stat1-dependent signaling inhibition in CF cells. The effect of elevated PIAS1 levels is functionally manifested in the presence of IFN- $\gamma$ by a demonstrable attenuation of Stat1-mediated luciferase expression through the pGAS-Luc construct in CF-phenotype 9/HTEo- pCEPRF cells, compared with control pCEP2 cells (Figure 8). We tested concentrations of IFN- $\gamma$ of 10,25 , and 100 units $/ \mathrm{mL}$, though the reported concentration of IFN- $\gamma$ in airway epithelial lining fluid is estimated to be less than 2 units/mL (31). It is important to note that pGAS-Luc reporter activity is stimulated in PCEPRF cells at higher IFN- $\gamma$ concentrations. As shown in Figure 5, Stat 1 protein levels are increased in CF cells. Higher IFN- $\gamma$ concentrations may alter the balance between PIAS1 and activated Stat1, allowing stimulation of the pGAS promoter. These data may point to a role for exogenous IFN- $\gamma$ as a possible therapeutic option in CF. Including this report, however, the expression of three proteins that are induced in response to IFN- $\gamma$, IL- $1 \beta$, or TNF- $\alpha$ have been shown to be diminished in CF epithelial cells: IRF-1, NOS2, and RANTES $(2-4,6)$. Although reduced RANTES expression in CF is reported to be caused by altered NF- $\kappa B$ activity (6), these three proteins have coregulatory mechanisms in common involving both NF- $\mathrm{KB}$-dependent and Stat1-dependent elements. One report demonstrates a physical interaction between NF- $\mathrm{\kappa B}$ and Stat 1 in promoter regulation when both of these regulatory elements are present (32). Increased binding of p-Stat 1 by PIAS1 may interrupt the necessary stoichiometry needed for the proper regulation of genes requiring both NF- $\mathrm{KB}$ and Stat 1 . This study demonstrates fundamental changes to a cell-signaling pathway that may have significant effects on disease progression in CF airways. 


\section{Acknowledgments}

This work was supported by a grant from the Cystic Fibrosis Foundation. The authors thank P. Davis for the 9/HTEo ${ }^{-}$pCEP2 and pCEPRF cells, B. Wible for informative discussion about KChAP/PIAS3, C. Garner for preparing tissue sections, and P. Bead for technical assistance.

1. Welsh, M.J., Tsui, L.C., Boat, T.F., and Beaudet, A.L. 1995. Cystic fibrosis. In The metabolic and molecular basis of inherited disease. 7 th edition. C.R. Scriver, A.L. Beaudet, W.S. Sly, and D. Valle, editors. McGraw-Hill. New York, New York. 3799-3863.

2. Meng, Q.-H., et al. 1998. Lack of inducible nitric oxide synthase in bronchial epithelium: a possible mechanism of susceptibility to infection in cystic fibrosis. J. Pathol. 184:323-331.

3. Kelley, T.J., and Drumm, M.L. 1998. Inducible nitric oxide synthase expression is reduced in cystic fibrosis murine and human airway epithelial cells. J. Clin. Invest. 102:1200-1207.

4. Elmer, H.L., Brady, K.G., Drumm, M.L., and Kelley, T.J. 1999. Nitric oxide-mediated regulation of transepithelial sodium and chloride transport in murine nasal epithelium. Am. J. Physiol. 276:L466-L473.

5. Kroesbergen, A., Jobsis, Q., Bel, E.H., Hop, W.C., and de Jongste, J.C. 1999. Flow-dependency of exhaled nitric oxide in children with asthma and cystic fibrosis. Eur. Respir. J. 14:871-875.

6. Schwiebert, L.M., Estell, K., and Propst, S.M. 1999. Chemokine expression in CF epithelia: implications for the role of CFTR in RANTES expression. Am. J. Physiol. 276:C700-C710.

7. Bryan, R., Kube, D., Perez, A., Davis, P.B., and Prince, A. 1998. Overproduction of the CFTR R domain leads to increased levels of asialoGM1 and increased Pseudomonas aeruginosa binding by epithelial cells. Am. J. Respir. Cell Mol. Biol. 19:269-277.

8. van Heeckeren, A., et al. 1997. Excessive inflammatory response of cystic fibrosis mice to bronchopulmonary infection with Pseudomonas aeruginosa. J. Clin. Invest. 100:2810-2815.

9. Tabary, O., et al. 1998. Selective up-regulation of chemokine IL-8 expression in cystic fibrosis bronchial gland cells in vivo and in vitro. Am. J. Pathol. 153:921-930.

10. Kammouni, W., Figarella, C., Marchand, S., and Merten, M. 1997. Altered cytokine production by cystic fibrosis tracheal gland serous cells. Infect. Immun. 65:5176-5183.

11. Chu, S.C., Marks-Konczalik, J., Wu, H.-P., Banks, T.C., and Moss, J. 1998. Analysis of the cytokine-stimulated human inducible nitric oxide synthase gene: characterization of differences between human and mouse iNOS promoters. Biochem. Biophys. Res. Commun. 248:871-878.

12. Marks-Konczalik, J., Chu, S.C., and Moss, J. 1998. Cytokine-mediated transcriptional induction of the human iNOS gene requires both activator protein 1 and nuclear factor kB-binding sites. J. Biol. Chem. 273:22201-22208.

13. Saura, M., Zaragoza, C., Bao, C., McMillan, A., and Lowenstein, C.J. 1999. Interaction of interferon regulatory factor- 1 and nuclear factor $\mathrm{kB}$ during activation of inducible nitric oxide synthase transcription. J. Mol. Biol. 289:459-471.

14. Salkowski, C.A., Barber, S.A., Detore, G.R., and Vogel, S.N. 1996. Differential dysregulation of nitric oxide production in macrophages with tar- geted disruptions in IFN regulatory factor-1 and -2 genes. J. Immunol. 156:3107-3110

15. Fujimura, M., et al. 1997. Attenuation of nitric oxide synthase induction in IRF-1-deficient glial cells. Brain Res. 759:247-250.

16. Heitmeier, M.R., Scarim, A.L., and Corbett, J.A. 1999. Prolonged STAT1 activation is associated with interferon-gamma priming for interleukin1-induced inducible nitric-oxide synthase expression by islets of Langerhans. J. Biol. Chem. 274:29266-29273.

17. Guo, F.H., et al. 1997. Interferon gamma and interleukin 4 stimulate prolonged expression of inducible nitric oxide synthase in human airway epithelium through synthesis of soluble mediators. J. Clin. Invest. 100:829-838.

18. Gao, J., Morrison, D.C., Parmely, T.J., Russell, S.W., and Murphy, W.J. 1997. An interferon-gamma-activated site (GAS) is necessary for full expression of the mouse iNOS gene in response to interferon-gamma and lipopolysaccharide. J. Biol. Chem. 272:1226-1230.

19. Wang, Y., O’Neal, K.D., and Yu-Lee, L. 1997. Multiple prolactin (PRL) receptor cytoplasmic residues and Stat 1 mediate PRL signaling to the interferon regulatory factor-1 promoter. Mol. Endocrinol. 11:1353-1364.

20. Li, X., Leung, S., Qureshi, S., Darnell, J.E., and Stark, G.R. 1996. Formation of STAT1-STAT2 heterodimers and their role in the activation of IRF-1 gene transcription by interferon-alpha. J. Biol. Chem. 271:5790-5794

21. Eckman, E., Cotton, C.U., Kube, D.M., and Davis, P.B. 1995. Dietary changes improve survival of CFTR S489X homozygous mutant mouse. Am. J. Physiol. 269:L625-L630.

22. Grubb, B.R., Vick, R.N., and Boucher, R.C. 1994. Hyperabsorption of Na and raised Ca-mediated chloride secretion in nasal epithelia of CF mice. Am. J. Physiol. 266:C1478-C1483.

23. Kelley, T.J., Thomas, K., Milgram, L.J.H., and Drumm, M.L. 1997. In vivo activation of the cystic fibrosis transmembrane conductance regulator mutant deltaF508 in murine nasal epithelium. Proc. Natl. Acad. Sci. USA. 94:2604-2608.

24. Kelley, T.J., Cotton, C.U., and Drumm, M.L. 1998. Regulation of amiloride-sensitive sodium absorption in murine airway epithelium by C-type natriuretic peptide. Am. J. Physiol. 274:L990-L996.

25. Perez, A., Risma, K.A., Eckman, E.A., and Davis, P.B. 1996. Overexpression of R domain eliminates cAMP-stimulated Cl- secretion in 9/HTEocells in culture. Am. J. Physiol. 217:L85-L92.

26. Steagall, W.K., Elmer, H.L., Brady, K.G., and Kelley, T.J. 2000. CFTRdependent regulation of epithelial NOS2 expression. Am. J. Respir. Cell Mol. Biol. 22:45-50.

27. Zhou, L., et al. 1994. Correction of lethal intestinal defect in a mouse model of cystic fibrosis by human CFTR. Science. 266:1705-1708.

28. Valdez, B.C., Henning, D., Perlaky, L., Busch, R.K., and Busch, H. 1997. Cloning and characterization of Gu/RH-II binding protein. Biochem. Biophys. Res. Commun. 234:335-340.

29. Liu, B., et al. 1998. Inhibition of Stat1-mediated gene activation by PIAS1. Proc. Natl. Acad. Sci. USA. 95:10626-10631.

30. Wible, B.A., Yang, Q., Kuryshev, Y.A., Accili, E.A., and Brown, A.M. 1998. Cloning and expression of a novel $\mathrm{K}+$ channel regulatory protein, KChAP. J. Biol. Chem. 273:11745-11751.

31. Jaffe, H.A., et al. 1991. Organ specific cytokine therapy. Local activation of mononuclear phagocytes by delivery of an aerosol of recombinant interferon-gamma to the human lung. J. Clin. Invest. 88:297-302.

32. Pine, R. 1997. Convergence of TNF-alpha and IFN-gamma signaling pathways through synergistic induction of IRF-1/ISGF-2 is mediated by a composite GAS/kB promoter element. Nucleic Acids Res. 25:4346-4354. 